\title{
Silicon Photonic Modulators for High Speed Optical Analog Links
}

\author{
(Invited) \\ C. Lacava ${ }^{1 *}$, D.J. Thomson ${ }^{1}$, K. Li ${ }^{1}$, I. Demirtzioglou ${ }^{1}$, A. Shakoor ${ }^{1}$, D. J. Richardson ${ }^{1}$, F. Zhang ${ }^{2}$, G. T. Reed ${ }^{1}$ \\ and P. Petropoulos ${ }^{1}$ \\ 1 - Optoelectronics Research Centre, University of Southampton, Southampton, Hampshire, UK. \\ 2 - State Key Laboratory of Advanced Optical Communication Systems and Networks, Peking University, Beijing, China. \\ *C.Lacava@soton.ac.uk
}

\begin{abstract}
We discuss recent progress on the design of silicon photonic modulators for high speed digital and analog optical links and their implementation using advanced modulation formats (including PAM and DMT).

OCIS codes: (230.4110) Modulators; (230.0250) Optoelectronics, (060.4510) Optical Communications
\end{abstract}

The field of silicon photonics has rapidly progressed in the last decade and a large amount of research has been performed both in academia and in the industry. A variety of devices have been demonstrated serving a wide range of applications, such as sensing, data communications and all-optical signal processing, showing remarkable performance [1]. The area of optical communications in particular, is key to silicon photonics and represents the major driver in advancing this technology. A number of key components for optical communications are now available in silicon photonics, including optical modulators, filters, receivers and optical signal processor devices $[1,2]$.

The optical intensity modulator represents one of the key components of an optical transmitter, thus a lot of efforts have been made in advancing the performance of this device [3]. Much research has focused on reducing the cost and power consumption of silicon modulators, while increasing their bandwidth at the same time, and components showing notable performance are now available to the community. On-off-keying (OOK)-operated devices exhibiting speeds that exceed $50 \mathrm{~Gb} / \mathrm{s}$ have been demonstrated with power consumption of a few pJ/bit. Advanced modulation formats can be used when operating these devices aiming to increase their spectral efficiency and enhance the device speed, reaching values that can exceed $100 \mathrm{~Gb} / \mathrm{s}$. Among the proposed solutions pulse amplitude modulation (PAM) and discrete multi-tone (DMT) formats have been recently identified as ideal candidates for developing the next generation of $100 \mathrm{~Gb} / \mathrm{s}$ silicon photonic-based direct-detection links [4,5].

Here we review our progress on the design and fabrication of silicon photonic modulators for high speed optical links. We show our recent results on PAM and DMT transmissions over unrepeatered optical links, demonstrating that 10G-class silicon photonic components can be used to generate high bit rate (50 Gb/s) signals with unprecedented spectral efficiencies, paving the way for the realization of low cost, compact direct-detection transceivers for short-reach applications (<100 km) exhibiting aggregate data rates in excess of $400 \mathrm{~Gb} / \mathrm{s}$.

[1] Soref, R. (2006). The Past , Present , and Future of Silicon Photonics. IEEE Journal of Selected Topics in Quantum Electronics, 12(6), 16781687.

[2] Lacava, C., Ettabib, M. A., \& Petropoulos, P. (2017). Nonlinear Silicon Photonic Signal Processing Devices for Future Optical Networks. Applied Sciences, 7(1), 103.

[3] Reed, G. T., Mashanovich, G., Gardes, F. Y., \& Thomson, D. J. (2010). Silicon optical modulators. Nature Photonics, 4.

[4] Dong, P., Lee, J., Chen, Y., Buhl, L. L., Chandrasekhar, S., Sinsky, J. H., \& Kim, K. (2015). Four-Channel 100-Gb / s per Channel Discrete Multi-Tone Modulation Using Silicon Photonic Integrated Circuits, 1(c), 4-6.

[5] Webster, M., Lakshmikumar, K., Appel, C., Muzio, C., Dama, B., \& Shastri, K. (2015). Low-Power MOS-Capacitor Based Silicon Photonic Modulators and CMOS Drivers. In Optical Fiber Communication Conference 2015 (Vol. 2, pp. 9-11). 